\title{
BMJ Open Sex differences in aneurysm morphologies and clinical outcomes in ruptured anterior communicating artery aneurysms: a retrospective study
}

\author{
Boli Lin, ${ }^{1,2}$ Weijian Chen, ${ }^{2}$ Lei Ruan, ${ }^{2}$ Yongchun Chen, ${ }^{2}$ Ming Zhong, ${ }^{3}$ \\ Qichuan Zhuge, ${ }^{1,3}$ Liang Hao Fan, ${ }^{3}$ Bing Zhao, ${ }^{3,4}$ Yunjun Yang ${ }^{1,2}$
}

To cite: Lin B, Chen W, Ruan L, et al. Sex differences in aneurysm morphologies and clinical outcomes in ruptured anterior communicating artery aneurysms: a retrospective study. BMJ Open 2016;6: e009920. doi:10.1136/ bmjopen-2015-009920

- Prepublication history for this paper is available online. To view these files please visit the journal online (http://dx.doi.org/10.1136/ bmjopen-2015-009920).

Received 10 September 2015 Revised 15 March 2016 Accepted 23 March 2016

\section{CrossMark}

\section{${ }^{1}$ Zhejiang Provincial Key Laboratory of Aging and Neurological Disorder Research, The First Affiliated Hospital, Wenzhou Medical University, Wenzhou, China 2Department of Radiology, The First Affiliated Hospital, Wenzhou Medical University, Wenzhou, China \\ ${ }^{3}$ Department of \\ Neurosurgery, The First \\ Affiliated Hospital, Wenzhou \\ Medical University, Wenzhou, China \\ ${ }^{4}$ Department of}

Neurosurgery, Mayo Clinic,

Rochester, Minnesota, USA

Correspondence to Dr Yunjun Yang or Dr Bing Zhao; wzfskyyj2011@163. com; drzhaobing@yahoo.com

\section{ABSTRACT}

Objectives: Ruptured anterior communicating artery $(A C O A)$ aneurysms occur more frequently in men. The purpose of the study was to investigate sex difference in aneurysm morphologies and clinical outcomes in patients with ruptured $A C O A$ aneurysms.

Setting: A tertiary referral hospital.

Participants: A total of 574 consecutive patients with ACoA aneurysms were admitted to our hospital from December 2007 to February 2015. In all, 474 patients (257 men and 217 women) with ruptured ACoA aneurysms were included in the study.

Main outcome measures: Aneurysm morphologies were measured using computed tomographic angiography and clinical outcomes were measured with Glasgow coma score at discharge.

Results: The aneurysm sizes $(p=0.001)$, aneurysm heights $(p=0.011)$, size ratios $(p<0.001)$, flow angles $(p=0.047)$ and vessel angles $(p=0.046)$ were larger in the male patients than in the females. The female patients more often had larger vessel sizes $(p=0.002)$. Multivariate logistic analysis revealed that significant differences in aneurysm morphologies between men and women were aneurysm size (OR $1.1,95 \% \mathrm{Cl} 1.0$ to $1.3 ; p=0.036)$, aneurysm height $(0 R 0.8,95 \% \mathrm{Cl}$ 0 . to $0.9 ; p=0.006$ ) and size ratio (OR $1.4,95 \% \mathrm{Cl} 0.5$ to $1.7 ; p=0.001$ ). There were no statistically significant differences in the outcomes between men and women (OR 1.0, 95\% Cl 0.6 to $1.7, p=0.857$ ).

Conclusions: The men were independently associated with larger aneurysm sizes, greater aneurysm heights and larger size ratios. Sex was not a risk factor for poor outcome in patients with ruptured $\mathrm{ACOA}$ aneurysms.

\section{INTRODUCTION}

Although aneurysmal subarachnoid haemorrhage (SAH) accounts for approximately 5\% of all strokes, it is a devastating condition with a high mortality and morbidity rate that leads to a high socioeconomic burden. ${ }^{1}$ Anterior communicating artery (ACoA)

\section{Strengths and limitations of this study}

- A large number of consecutive patients with ruptured anterior communicating artery (ACOA) aneurysms were analysed.

- The men were associated with larger aneurysm sizes, larger size ratios and greater aneurysm heights.

- Sex was not a risk factor for poor clinical outcome of ruptured ACoA aneurysms.

- This study was a single-centre retrospective analysis without long-term follow-up data.

aneurysms have a higher risk of rupture than aneurysms at other locations. ${ }^{2}$ However, the mechanisms of the formation and rupture of ACoA aneurysms are not well understood. Previous studies examined sex differences in aneurysmal SAHs and found that aneurysmal SAHs occur more frequently in women, whereas ruptured ACoA aneurysms occur more frequently in men. ${ }^{3-5}$ Additionally, women of comparable age fare worse than men with respect to morbidity and mortality after SAH. ${ }^{6}{ }^{7}$ However, there is little information about sex-specific differences in the outcomes of ruptured ACoA aneurysms.

Aneurysm morphologies such as aneurysm size and size ratio are important risk factors for the rupture of aneurysms..$^{8-11}$ We hypothesised that sex differences in aneurysm morphologies could result in the different prevalence of ruptured ACoA aneurysms between men and women. An extensive analysis of sex differences in patients with ruptured ACoA aneurysms may improve our understanding of the pathophysiology of ACoA aneurysm formation, growth and rupture.

In this study, we retrospectively reviewed the aneurysm morphologies and clinical outcomes in consecutive patients with ruptured 
ACoA aneurysms to identify the significant differences between men and women.

\section{METHODS}

Study design and patients

This study was approved by the Institutional Review Board of the Hospital. From December 2007 to February 2015, 574 consecutive patients with 584 ACoA aneurysms were admitted to our hospital. The details of patient cohort have been described previously. ${ }^{12}{ }^{13}$ We retrospectively collected the following data: sex, age, World Federation of Neurosurgical Societies (WFNS) grade at admission, Fisher grade, CT angiography (CTA) image data, treatment modalities (endovascular treatment, surgical treatment or medical treatment), angiographic vasospasm on initial CTA, cerebral infarction and Glasgow Outcome Scale (GOS) at discharge. Poor outcome was defined as a GOS of 1, 2 or 3. Patients with SAH caused by the rupture of an ACoA aneurysm were included in this study. Patients with fusiform aneurysms, two ACoA aneurysms, and patients whose images were of poor quality and could not be used to measure the aneurysm morphologies, were excluded.

\section{Reconstruction of CTA images}

A 16-channel multidetector CT scanner (Lightspeed pro 16; General Electric Medical Systems, Milwaukee, Wisconsin, USA), a 64-channel multidetector CT scanner (Lightspeed VCT 64 General Electric Medical Systems, Milwaukee, Wisconsin, USA) and a 320-detector row CT scanner (Aquilion ONE, Toshiba Medical Systems, Japan) were used to acquire the CTA images. ${ }^{12}$ A V.4.6 (GE Medical Systems) workstation was used to reconstruct the three dimensional images of the aneurysms and surrounding vasculatures, and to measure the sizes, heights and angles of the aneurysms or vasculatures. All measurements were performed by two independent neuroradiologists who were blinded to the sex of the patients.

\section{Aneurysm morphology and definition}

The morphological parameters, including vessel size, aneurysm size, aneurysm height, perpendicular height, neck size, size ratio, aspect ratio, flow angle, vessel angle and aneurysm angle, have been described previously. ${ }^{12} 13$ Aneurysm size was the largest cross-sectional diameter of the aneurysm. Aneurysm height was measured between the centre of the aneurysm neck and the greatest distance to the aneurysm dome. Vessel size was the mean vessel diameter of all arteries associated with the aneurysm. The vessel diameter of a particular artery was determined by averaging the diameter of the cross-section of the vessel at the neck of the aneurysm (D1) and the diameter of the cross-section at $1.5 \times \mathrm{D} 1$ distance from the neck of the aneurysm. Size ratio was the ratio between the aneurysm height and vessel size. The aneurysm morphologies measurements are illustrated in figure 1 . The average values were used for the analysis.

\section{Statistical analysis}

The statistical analyses were performed using IBM SPSS V.22.0 (IBM SPSS, Armonk, New York, USA). The aneurysm characteristics were compared between the male and female groups. Independent-samples $t$ tests and Mantel-Haenszel tests were used for the continuous variables, and $\chi^{2}$ tests and Fisher's exact tests were used for the categorical variables. All variables with $\mathrm{p}$ values $<0.10$ in the univariate analyses were entered into the multivariate analysis model. The first multivariate model was used to identify significant differences in the aneurysm characteristics between the men and women using the backward logistic regression method. The second multivariate model was used to assess the sex differences in the outcomes using an enter logistic regression method after adjustments for the potential confounders, as appropriate. The third model was used to determine the independent risk factors for poor outcomes in the patients who were treated with endovascular or surgical treatments. The ORs and 95\% CIs were calculated. $p$ Values $<0.05$ were considered statistically significant.
Figure 1 Aneurysm

morphologies measurements on CT angiography. (A) Aneurysm geometry; (B) Aneurysm vessels; Hmax, aneurysm height; Dmax, aneurysm size; $\mathrm{Ph}$, perpendicular height; N, neck size; D1 and D2, cross-section diameter of the artery.

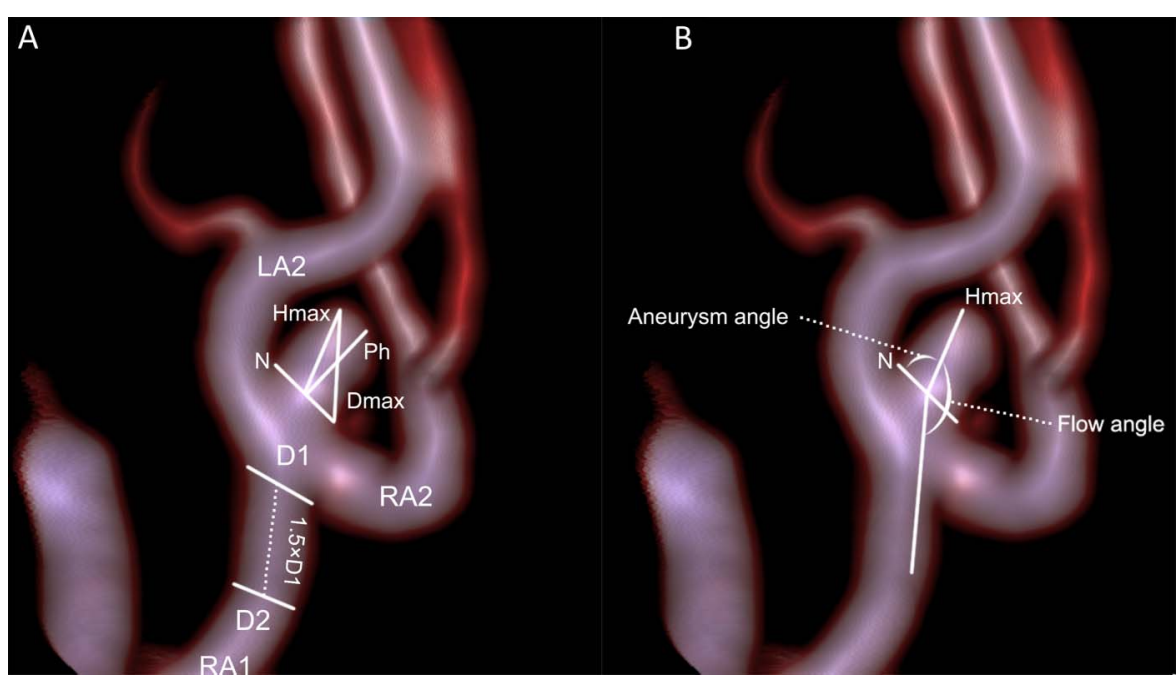




\section{RESULTS}

\section{Clinical characteristics}

Of the 574 patients, 100 patients were excluded, and these included 45 patients with unruptured aneurysms, 38 patients whose CTA images were of poor quality, 7 patients with fusiform aneurysms and 10 patients with 2 ACoA aneurysms. Ultimately, 474 patients were included in this study (257 men and 217 women). The demographic and clinical characteristics are provided in table 1 . The female patients were significantly older than the male patients (mean ages of 57.5 and 54.1 years, respectively, $\mathrm{p}=0.002$ ). The distributions of the WFNS grades and Fisher grades were similar between the genders. There were no statistically significant differences in the treatment modalities, angiographic vasospasms or cerebral infarctions between the male and female patients.

\section{Aneurysm morphologies}

The morphological characteristics of the ACoA aneurysms are presented in table 1 . The aneurysm size (5.6 vs
$4.9 \mathrm{~mm}$, respectively, $\mathrm{p}=0.001)$, aneurysm heights (4.5 vs $4.0 \mathrm{~mm}$, respectively, $\mathrm{p}=0.011$ ), size ratio (2.9 vs 2.1 , $\mathrm{p}<0.001)$ flow angles $\left(137.0^{\circ}\right.$ vs $132.1^{\circ}$, respectively, $\mathrm{p}=0.047)$ and vessel angles $\left(62.5^{\circ}\right.$ vs $57.5^{\circ}$, respectively, $\mathrm{p}=0.046$ ) were larger in the male patients than in the female patients. The female patients more often had larger vessel sizes compared with the male patients (2.0 vs $1.8 \mathrm{~mm}$, respectively, $\mathrm{p}=0.002$ ). The multivariate logistic regression analysis revealed that the significant sex differences in aneurysm morphologies were the aneurysm size $(p=0.036)$, aneurysm height $(p=0.006)$ and size ratio $(\mathrm{p}=0.001)$ (table 2$)$.

\section{Clinical outcomes}

At discharge, 24 (13.1\%) of 183 female patients and 27 $(12.3 \%)$ of 220 male patients had a poor outcome after endovascular or surgical treatment. The outcomes at discharge for all of the patients and those who received aggressive treatment are presented in figure 2. Among the patients who were treated with endovascular coiling

Table 1 Sex differences in clinical characteristics and aneurysm morphologies

\begin{tabular}{|c|c|c|c|}
\hline Characteristics & Men $(n=257)$ & Women $(n=217)$ & p Value \\
\hline Age (years) & $54.1 \pm 11.6$ & $57.5 \pm 12.8$ & 0.002 \\
\hline WFNS grade at admission & $1.7 \pm 1.3$ & $1.6 \pm 1.3$ & 0.692 \\
\hline WFNS grade I-II & $204(79.8 \%)$ & $178(82.4 \%)$ & \\
\hline WFNS grade III & $7(2.7 \%)$ & $4(1.9 \%)$ & \\
\hline WFNS grade IV-V & $45(17.5 \%)$ & $34(15.7 \%)$ & \\
\hline Fisher grade at admission & & & 0.380 \\
\hline Grade I-II & $34(13.2 \%)$ & $26(12.0 \%)$ & \\
\hline Grade III & $80(31.1 \%)$ & $55(25.2 \%)$ & \\
\hline Grade IV & $123(47.9 \%)$ & $121(55.8 \%)$ & \\
\hline Missing & $20(7.8 \%)$ & $15(7.0 \%)$ & \\
\hline \multicolumn{4}{|l|}{ Aneurysm morphology } \\
\hline Vessel size $(\mathrm{mm})$ & $1.8 \pm 0.6$ & $2.0 \pm 0.5$ & 0.002 \\
\hline Aneurysm size (mm) & $5.6 \pm 2.6$ & $4.9 \pm 2.3$ & 0.001 \\
\hline Aneurysm height (mm) & $4.5 \pm 2.2$ & $4.0 \pm 2.1$ & 0.011 \\
\hline Perpendicular height (mm) & $3.7 \pm 1.8$ & $3.4 \pm 1.8$ & 0.107 \\
\hline Neck size $(\mathrm{mm})$ & $3.0 \pm 1.1$ & $2.9 \pm 1.2$ & 0.245 \\
\hline Size ratio & $2.9 \pm 2.7$ & $2.1 \pm 1.3$ & $<0.001$ \\
\hline Aspect ratio & $1.3 \pm 0.8$ & $1.2 \pm 0.6$ & 0.306 \\
\hline Flow angle & $137.0 \pm 27.9$ & $132.1 \pm 27.3$ & 0.047 \\
\hline Vessel angle & $62.5 \pm 26.5$ & $57.5 \pm 28.4$ & 0.046 \\
\hline Aneurysm angle & $70.1 \pm 19.4$ & $70.9 \pm 17.4$ & 0.609 \\
\hline Aneurysm projection & & & 0.858 \\
\hline Anterior projection & $189(73.5 \%)$ & $158(72.8 \%)$ & \\
\hline Posterior projection & $68(26.5 \%)$ & $59(27.2 \%)$ & \\
\hline A1 segment configuration & & & 0.497 \\
\hline Symmetric & 77 (30.0\%) & $76(35.0 \%)$ & \\
\hline Dominant & $115(44.7 \%)$ & $89(41.0 \%)$ & \\
\hline Complete & $65(25.3 \%)$ & $52(24.0 \%)$ & \\
\hline Multiple aneurysms & $31(12.1 \%)$ & $38(17.6 \%)$ & 0.094 \\
\hline Treatment modality & & & 0.883 \\
\hline Clipping & 96 (37.4\%) & 77 (35.5\%) & \\
\hline Coiling & $124(48.2 \%)$ & $106(48.8 \%)$ & \\
\hline Medical treatment & $37(14.4 \%)$ & $34(15.7 \%)$ & \\
\hline Angiographic vasospasm & $21(8.2 \%)$ & $24(11.1 \%)$ & 0.291 \\
\hline Cerebral infarction & $24(9.3 \%)$ & $25(11.5 \%)$ & 0.437 \\
\hline
\end{tabular}


Table 2 Multivariate analyses of aneurysm characteristics in men compared with women

\begin{tabular}{lll}
\hline Variables & OR $95 \% \mathbf{C l}$ & p Value \\
\hline Aneurysm size & $1.1(1.0$ to 1.3$)$ & 0.036 \\
Aneurysm height & $0.8(0.6$ to 0.9$)$ & 0.006 \\
Size ratio & $1.4(1.2$ to 1.7$)$ & 0.001 \\
\hline
\end{tabular}

or surgical clipping, there were no statistically significant differences of poor outcomes between the male and female patients after adjusting for age, aneurysm size, vessel size, aneurysm height, size ratio, flow angle and vessel angle (OR 1.0, 95\% CI 0.6 to $1.7, \mathrm{p}=0.857$ ). The univariate and multivariate analyses for predictors of poor outcome among the treated patients are presented in table 3. Older patients $(\mathrm{p}<0.001)$, greater aneurysm height $(p=0.040)$ and perpendicular height $(p=0.011)$, higher Fisher grade $(p=0.002)$, higher WFNS grade $(\mathrm{p}<0.001)$ and cerebral infarction $(\mathrm{p}=0.001)$, were associated with poor clinical outcomes at discharge. The multivariate analysis revealed that older age $(\mathrm{p}=0.002)$ and higher WFNS grade at admission $(p<0.001)$ were independently associated with poor outcomes.

\section{DISCUSSION}

In this study, 257 men and 217 women presented with ruptured ACoA aneurysms, with female-to-male ratio of $0.8: 1$. Our results are consistent with a previous study showing that women exhibited a lower rate of ruptured ACoA aneurysms. ${ }^{4}$ The significant sex differences in aneurysm morphologies were aneurysm size, aneurysm height and size ratio. The men more often had larger aneurysms and larger size ratios. These results suggest that the mechanisms of ACoA aneurysm formation, growth and rupture, may differ between men and women, and may aid in the understanding of the high incidence of ruptured ACoA aneurysms in men. In addition, there were no significant differences in the severity of bleeding or the outcomes between the women and men.

\section{Sex difference of risk of ACOA aneurysms}

Ruptured ACoA aneurysms more often occur in men than in women. Sex differences in the occurrence of ACoA aneurysms have been reported in the previous studies. ${ }^{4} 7^{14}$ Aarhus et $a l^{3}$ compared unruptured and ruptured aneurysms with respect to anatomical location, based on a stable population in a defined geographical region, and demonstrated that ACoA aneurysms had a higher rupture risk and that ruptured ACoA aneurysms are over-represented among men. Our findings are consistent with their studies.

\section{Sex difference of aneurysm methodologies}

Sex difference in the occurrence of ruptured ACoA aneurysms may be explained by the sex-linked differences in the aneurysm morphologies. We found that the aneurysm size in men was larger than that in women. These results are in line with other studies showing that male patients had larger ruptured aneurysms than female patients. ${ }^{15-17}$ Although aneurysm size is a wellknown risk factor for aneurysm rupture, we found that the size ratio was greater than the significance of any of the other morphologies, including aneurysm size and aneurysm height.

The size ratio captures the maximum shape deformation of the parent vessel. Kashiwazaki and Kuroda ${ }^{18}$ reported the relationship of the size ratio to the rupture in small intracranial aneurysms and found that the size ratio was an independent risk factor associated with aneurysm rupture. Increases in the size ratio result in more complex intra-aneurysmal flow patterns, multiple vortices and increased areas of low aneurysmal wall shear stress. ${ }^{9} 19$ Complex flow patterns and instability of the flow also increase the aneurysm size, aneurysm height and the size ratio. ${ }^{12}$ We also found that male sex was associated with greater aneurysm heights. These results were consistent with larger size ratios.

\section{Sex difference of clinical outcomes}

Female sex is thought to be a risk factor for poor outcome following aneurysmal SAH. ${ }^{720}$ Rosenørn et $a l^{7}$ reported 1076 patients with aneurysmal SAH (674
Figure 2 Clinical outcomes in patients with ruptured anterior communicating artery aneurysms by sex. (A) Outcomes in all patients. (B) Outcomes in patients treated with endovascular coiling or surgical clipping. GOS, Glasgow Outcome Score.
A

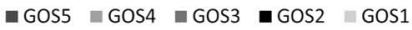

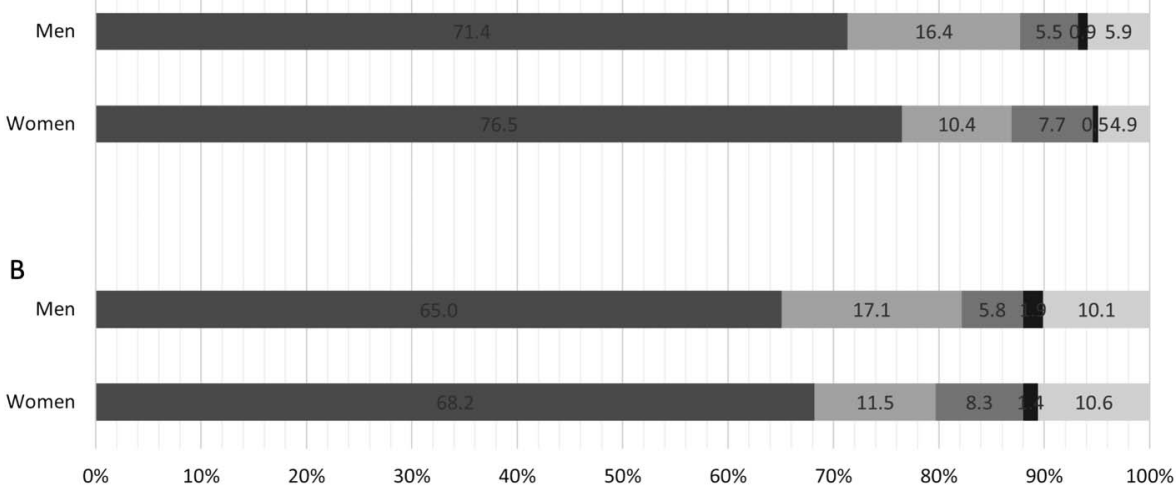


Table 3 Univariate and multivariate analyses for predictors of poor outcome

\begin{tabular}{|c|c|c|c|c|}
\hline \multirow[b]{2}{*}{ Variables } & \multicolumn{2}{|c|}{ Unadjusted analysis } & \multicolumn{2}{|c|}{ Multivariate analysis } \\
\hline & OR (95\% Cl) & p Value & OR (95\% Cl) & p Value \\
\hline Age & 1.1 (1.0 to 1.1$)$ & $<0.001$ & $1.1(1.0$ to 1.1$)$ & 0.002 \\
\hline Aneurysm height & 1.1 (1.0 to 1.3$)$ & 0.040 & & \\
\hline Perpendicular height & $1.2(1.0$ to 1.4$)$ & 0.011 & & \\
\hline Fisher grade & 1.9 (1.3 to 2.9$)$ & 0.002 & & \\
\hline WFNS grade & 2.3 (1.9 to 2.8$)$ & $<0.001$ & 2.3 (1.9 to 2.8$)$ & $<0.001$ \\
\hline Treatment modality & 1.7 (1.0 to 3.1$)$ & 0.067 & & \\
\hline Cerebral infraction & 3.3 (1.6 to 6.8$)$ & 0.001 & & \\
\hline
\end{tabular}

women and 402 men) and found significantly worse outcomes in females than in males, with increased incidence of angiographic vasospasm in females. In our study, we did not identify greater risks of vasospasms on the initial CTAs, nor of cerebral infarctions, in women compared with in men. Moreover, the outcomes at discharge were similar between the genders in both, the univariate analysis and the multivariate logistic regression analyses. The multivariate analysis of the predictors of poor outcome also showed sex was not an independent risk factor for poor clinical outcome except for age and WFNS grade at admission. Our results are in agreement with those of several previous studies on the sex differences in the outcomes of $\mathrm{SAH},{ }^{4}{ }^{21}$ in which the rates of vasospasm were also found to be similar between men and women, and the probabilities of good outcomes at 3 months were virtually equivalent in women and men after adjusting for age. The lack of sex differences in the outcomes was probably due to the advances in surgical and endovascular treatments for ruptured aneurysms.

\section{Limitations}

This study has several limitations. First, this study was a single-centre retrospective. The majority of patients presented with ruptured aneurysms, and patients with poor quality images that could not be well reconstructed to measure aneurysm morphologies were excluded; therefore, it is difficult to avoid selection bias. Second, other potential risk factors for the occurrence and rupture of ACoA aneurysms except for aneurysm morphologies were not defined. We focused on sex differences in aneurysm morphologies and clinical outcomes. Third, we analysed patients who were admitted alive with SAH caused by a ruptured ACoA aneurysm. These conclusions may apply to survivors of ruptured aneurysms. However, gender analysis of the fraction of patients who died before arrival is impossible. With the improvement of patient referral and ambulance systems, almost all patients with SAH can arrive in the hospital to get further treatment. Finally, the long-term outcomes were not evaluated in this study. However, short-term outcomes are associated with long-term outcomes after treatment because the 2-month outcomes are predictive of the 1-year outcomes. ${ }^{22}$ Nevertheless, we analysed a large number of consecutive patients with ruptured ACoA aneurysms and found that significant sex differences in aneurysm morphologies were the aneurysm size, aneurysm height and the size ratio.

\section{CONCLUSIONS}

In a large number of patients with ruptured ACoA aneurysms, the male patients were more likely to exhibit larger aneurysm sizes, greater aneurysm heights and larger size ratios. These results may help explain the higher proportion of men with ruptured ACoA aneurysms. Due to advances in the treatment of ruptured aneurysms, sex may not be a risk factor for poor outcomes of ruptured ACoA aneurysms.

Contributors $\mathrm{BL}, \mathrm{LR}, \mathrm{YC}$ and $\mathrm{LHF}$ were involved in data measurement, collection and verification. WC, MZ and QZ were involved in data interpretation. BZ was involved in data analysis. BL drafted the manuscript. BZ and $Y Y$ were involved in the study design and revision of the manuscript. All the authors read and approved the final manuscript.

Funding This work was supported by Zhejiang Provincial Key Laboratory of Aging and Neurological Disorder Research (grant 2012E10008) and Wenzhou public welfare planning project of science and technology (grant Y20140731).

Competing interests None declared.

Patient consent Obtained.

Ethics approval The study was approved by the Institutional Review Board of the first affiliated hospital of Wenzhou Medical University.

Provenance and peer review Not commissioned; externally peer reviewed.

Data sharing statement No additional data are available.

Open Access This is an Open Access article distributed in accordance with the Creative Commons Attribution Non Commercial (CC BY-NC 4.0) license, which permits others to distribute, remix, adapt, build upon this work noncommercially, and license their derivative works on different terms, provided the original work is properly cited and the use is non-commercial. See: http:// creativecommons.org/licenses/by-nc/4.0/

\section{REFERENCES}

1. Rivero-Arias O, Gray A, Wolstenholme J. Burden of disease and costs of aneurysmal subarachnoid haemorrhage (aSAH) in the United Kingdom. Cost Eff Resour Alloc 2010;8:6.

2. Forget TR Jr, Benitez R, Veznedaroglu $\mathrm{E}$, et al. A review of size and location of ruptured intracranial aneurysms. Neurosurgery 2001;49:1322-5; discussion 25-6.

3. Aarhus M, Helland CA, Wester K. Differences in anatomical distribution, gender, and sidedness between ruptured and 
unruptured intracranial aneurysms in a defined patient population. Acta Neurochir (Wien) 2009;151:1569-74.

4. Hamdan A, Barnes J, Mitchell P. Subarachnoid hemorrhage and the female sex: analysis of risk factors, aneurysm characteristics, and outcomes. J Neurosurg 2014;121:1367-73.

5. Kongable GL, Lanzino G, Germanson TP, et al. Gender-related differences in aneurysmal subarachnoid hemorrhage. J Neurosurg 1996;84:43-8.

6. Pekmezovic T, Tepavcevic DK, Jarebinski M, et al. Trends in mortality from different subtypes of stroke in the population of Belgrade (Serbia). Clin Neurol Neurosurg 2008;110:51-7.

7. Rosenørn J, Eskesen V, Schmidt K. Clinical features and outcome in females and males with ruptured intracranial saccular aneurysms. Br J Neurosurg 1993;7:287-90.

8. Dhar S, Tremmel M, Mocco J, et al. Morphology parameters for intracranial aneurysm rupture risk assessment. Neurosurgery 2008;63:185-96; discussion 196-7.

9. Tremmel M, Dhar S, Levy El, et al. Influence of intracranial aneurysm-to-parent vessel size ratio on hemodynamics and implication for rupture: results from a virtual experimental study. Neurosurgery 2009;64:622-30; discussion 30-1.

10. Orz $\mathrm{Y}$, Kobayashi $\mathrm{S}$, Osawa M, et al. Aneurysm size: a prognostic factor for rupture. Br J Neurosurg 1997;11:144-9.

11. Beck J, Rohde S, Berkefeld J, et al. Size and location of ruptured and unruptured intracranial aneurysms measured by 3-dimensional rotational angiography. Surg Neurol 2006;65:18-25; discussion 25-7.

12. Xia N, Liu Y, Zhong M, et al. Smoking associated with increased aneurysm size in patients with anterior communicating artery aneurysms. World Neurosurg 2016;87:155-61.

13. Shao $\mathrm{X}$, Wang $\mathrm{H}$, Wang $\mathrm{Y}$, et al. The effect of anterior projection of aneurysm dome on the rupture of anterior communicating artery aneurysms compared with posterior projection. Clin Neurol Neurosurg 2016;143:99-103.

14. Lindner SH, Bor AS, Rinkel GJ. Differences in risk factors according to the site of intracranial aneurysms. J Neurol Neurosurg Psychiatry 2010;81:116-18.

15. Liu $\mathrm{H}$, Zhang $\mathrm{T}$, Jiao $\mathrm{S}$, et al. Epidemiological investigation of 264 sporadic cases of ruptured cerebral aneurysm at a single institution in southwest China. Neuropsychiatr Dis Treat 2015;11: 1609-14.

16. Zhao L, Zhang L, Zhang X, et al. An analysis of 1256 cases of sporadic ruptured cerebral aneurysm in a single Chinese institution. PLOS ONE 2014; 9:e85668.

17. Ho AL, Lin N, Frerichs $\mathrm{KU}$, et al. Smoking and intracranial aneurysm morphology. Neurosurgery 2015;77:59-66.

18. Kashiwazaki D, Kuroda S. Size ratio can highly predict rupture risk in intracranial small $(<5 \mathrm{~mm})$ aneurysms. Stroke 2013;44:2169-73.

19. Xiang J, Natarajan SK, Tremmel M, et al. Hemodynamicmorphologic discriminants for intracranial aneurysm rupture. Stroke 2011;42:144-52.

20. George B, Zerah M, Mourier KL, et al. Ruptured intracranial aneurysms. The influence of sex and fibromuscular dysplasia upon prognosis. Acta Neurochir (Wien) 1989;97:26-30.

21. Park SK, Kim JM, Kim JH, et al. Aneurysmal subarachnoid hemorrhage in young adults: a gender comparison study. J Clin Neurosci 2008;15:389-92.

22. Molyneux AJ, Kerr RS, Yu LM, et al. International subarachnoid aneurysm trial (ISAT) of neurosurgical clipping versus endovascular coiling in 2143 patients with ruptured intracranial aneurysms: a randomised comparison of effects on survival, dependency, seizures, rebleeding, subgroups, and aneurysm occlusion. Lancet 2005;366:809-17. 